\title{
Pragmatic Interoperability for Extreme Automation and Healthcare Interoperability \& Continuity
}

\author{
Jinan Fiaidhi', Senior Member, IEEE and Sabah Mohammed ${ }^{1}$, Senior Member, IEEE \\ ${ }^{1}$ Department of Computer Science, Smart Health FabLab, Lakehead University, Thunder Bay, Ontario P7B 5E1, CANADA \\ Corresponding author: Jinan Fiaidhi (e-mail: jfiaidhi@lakeheadu.ca).
}

\begin{abstract}
Today's world is wired differently compared to the past few years where almost everything can be made ubiquitously with high degree of connectivity. Such ecosystem of ubiquitous connectivity is a critical piece for the advancements of key industries like manufacturing and healthcare. Both of these industries are transitioning their delivery system from volume to value and are looking for new ways to maintain continuity, cut costs, improve quality and increase the levels of interoperability. Accomplishing these goals will help to create a seamless workflow and build a foundation to advance value-based industry. However, manufacturing and healthcare organizations today are balancing the complexities of the using innovative digital transformation technologies with the fusion of working with a multi-disciplinary working teams, designers, developers and customers to achieve enterprise resilience aligned to fiscal and fiduciary responsibility, customer commitments and values, regulatory and compliance requirements and stakeholders' expectations. To integrate such diverse range of perspectives and turn those into meaningful insights requires the planning and enforcement of "Pragmatic Interoperability". This paper describes the author's vision in developing a flexible workflow infrastructure for enforcing the pragmatic interoperability in industries like manufacturing and healthcare. This vision is based on business continuity planning, web services interoperability, Node-Red and IFTTT workflow technologies.
\end{abstract}

INDEX TERMS Extreme Automation, Web Services, Services Workflow Composition, Node-red, IFTTT technologies, Business Continuity Planning

\section{INTRODUCTION}

Many industries learn the lesson of dealing with the complexity of distributed fusion systems and employ variety of advanced techniques, which involve examining processes end to end, and identifying and eliminating waste and reducing risks [1]. These advanced techniques include technologies like:

$>$ Just-in-time (JIT) systems,

$>$ Computer integrated manufacturing (CIM),

$>$ Concurrent engineering $(\mathrm{CE})$,

$>$ Flexible manufacturing systems (FMS)

$>$ Collaborative manufacturing $(\mathrm{CM})$

$>$ Meaningful Use (MU)

$>$ Multisensory Data Fusion (MSDF)

to produce products and services with high quality, low costs and shorter lead times [2]. However, enterprise collaboration must involve new type of human engagement, involvement and collaboration as well as proactive planning process that ensures critical services or products are going to be delivered during a disruption. Although the transformation towards such socio technical ecosystem is underway to boost productivity, minimizing risks, simplifying human jobs and ultimately accelerating the industrial growth across geographies, the challenges remain are huge as this integration involves a fusion of technologies that is "blurring the lines between the physical, digital, and human spheres" [3]. It is set to disrupt people, society, business, and government through its innovations. The new face of industry needs to be defined by a confluence between technical complexity and the social system in which the technical complexity is embedded. This new face includes an ecosystem of extreme connectivity, extreme automation, extreme environments, cloud computing delivery, smart digital devices, human and machine collaborations and services interoperability. In the paradigm of manufacturing, this type of ecosystem is focused on using the notion of 'Smart Factory' enriched with capabilities to encourage interoperability and collaboration including [4]:

1. Electronic Kanban's instead of Legacy Production Orders

2. Production Surveillance and Prediction

3. Total Productive Maintenance

4. Real Time Data Analysis

5. Virtual Value Stream Mapping

However, researchers from the paradigm of organizational semiotics (OS) criticized this approach as it is lacking the 
social level $[5,6,7]$. To develop a validated OS research model, a two-stage research design need to be adopted including an assessment study and planning study. The assessment study confirms factors identified from existing experiences as well as to explore any new important factors from empirical and experience context based on sound techniques like:

$>$ Situational Assessment and Risks Analysis

$>$ Business Impact Analysis

$>$ Disaster Recovery Investigation

$>$ Fusion Interoperability Mocking

The assessment study constructs the industry model based on a top-down black-box approach, which organizes the observations of the operational systems into structures of agents and affordances interlinked by ontological dependencies and governed by metadata norms. The planning study is to validate the resilience of this model in order to ensure the presentation of a reliable and coherent picture to the stakeholders as well as to fuse data streams and services in real-time, issuing alerts when anomalous behavior or a disaster is detected and to mine the raw data for patterns that represent information and storing the set of patterns in the knowledge base that is maintained by the system. Figure 1 illustrates the overall idea of semiotic paradigm in developing an integral model in top-down way starting from Part A and ending with Part C.

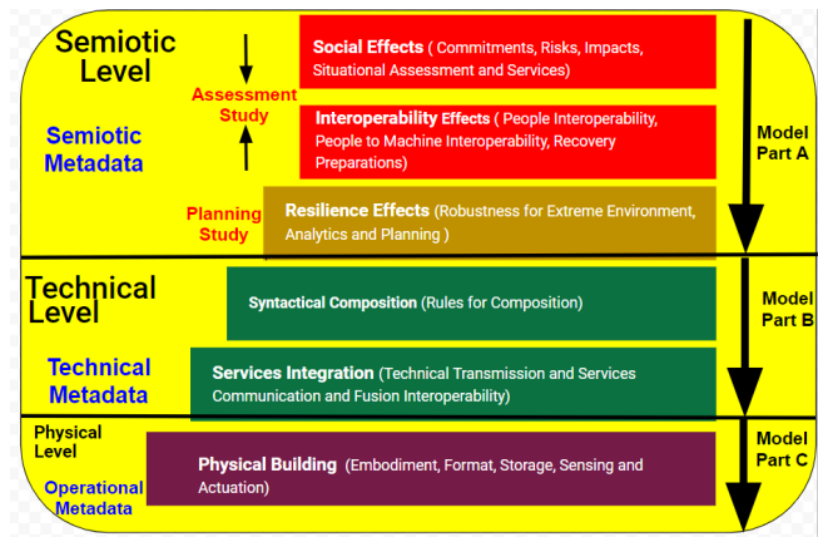

Figure 1: A Top-Down Organizational Semiotic Framework for Designing Sociotechnical Systems.

In the semiotic model, metadata information is indispensable for data sharing and interoperability in all the parts of the system model, however, only rarely are metadata available or can be designed in a structured, comprehensive, and machine-readable form. This poses a severe technical challenge for finding and retrieving data as well as for the integrating the various components of the sociotechnical system. The downside of the semiotic "top-down" approach in which authorities defines the "standard," of collaboration which is not flexible and open enough and will inevitably lag behind the ever-changing requirements due to the dynamics in developing sociotechnical systems. Furthermore, this "standard" would need to be accepted by the collaborating parties and components.

Several other researchers developed more dynamic models to deal with interoperability and fusion integration. The models eliminate the need for separate modules across the many areas of risk and continuity management, making it easy to expand into new areas when ready, operating as a completely integrated program on a common information foundation. In this direction there are four models that have been taken as a lingua franca for data fusion problems including:

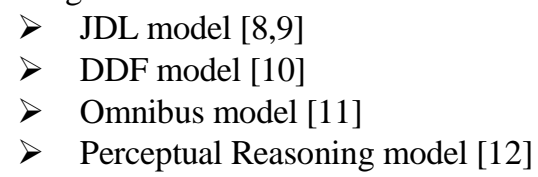

However, none of these models deal with multiple data of different types as well as account for human processing. All these models perform automatic processing of signals from machines and does not account for human interactions. In a typically system architecture one need to support the role and interactions of users as well as fusion of components and services. This paper proposes an extension to JDL Model where it can support different types of data integration and user interactions as intrinsic part of the fusion system for resilience business and continuity.

\section{Pragmatic Interoperability for Extreme Automation and Healthcare Interoperability and Continuity}

Pragmatic interoperability has been highlighted as a key requirement to enhance collaboration [13]. However, fulfilling this requirement is not a trivial task and there is a lack of works discussing solutions to achieve this level of interoperability for extreme automation as applied to key industries like manufacturing and healthcare. The interoperability of enterprises is a challenging goal that could be achieved by different approaches and among them the federated ones where different enterprises can inter operate without any particular effort and where the dynamism and the autonomy aspects are supported. The Joint Directorate Laboratories (JDL) model addresses this challenge, which was researched in 1993 to deal with multi-level data fusion interoperability and integration, primarily was for military systems [14]. The idea was that you could look at the state of the world considering different entities as "atomic units" of your "World View". Level 1 Fusion, for example, was called "Object Refinement" and was focused on fusing multiple heterogeneous data sources to obtain information about individual objects (e.g., people, vehicles, buildings, etc.). Socalled Higher-Level Fusion dealt with Levels 2-4, which were named "Situation Refinement", "Threat Refinement", and "Process Refinement". Note, there is a big conceptual jump from Level 1 to Level 2. Level 1 consists of real, measurable objects that exist in time and space. The other levels are concepts and exist in "linguistic space" instead of 
physical space. In 1998, the JDL model was upgraded to more general, less militaristic language. There was also a "Level 0" added. So the levels now were "Sub-Object Assessment", "Object Assessment", "Situational Assessment", "Impact Assessment", and "Process Refinement". Sub-Object Assessment referred to using data to resolve things smaller than individual objects. So it could be an arm, or it could refer to integrating ("fusing") consecutive reflected pulses from a radar to form a signal [15]. In 2015, a researcher [16], extended the four levels JDL model into five level one where Level 5: "User Refinement" involves humans to be "in the loop" affecting the products of all the lower levels.

However, the increasing amount of information available for business planning motivates the adoption of machine learning methods for addressing specific situation assessment or risk. A special focus needs to be placed on prognosis, namely, the capability to estimate and anticipate events of interest regarding assets and production processes. There lies indeed at the core challenge of business modeling and planning from a data science perspective: data-driven prognostic approaches aim at predicting when an abnormal behavior is likely to arise within the monitored process, providing further insights such as its severity and impact on the business performance. For this reason, it becomes particularly interesting to characterize normality properly towards unveiling degradation patterns or trends. Thus, adding a sixth level to JDL to include thick data and machine learning analytics is one of the most important direction for business modeling and planning. The idea of having the sixth level is to characterize the business values (including user values) through qualitative measures (aka Thick Data [17]) as well as to identify behavioral patterns of interest on the basis of the data monitored from the process or asset under study (training data) by means of quantitative analytics and machine learning models. This acquired knowledge (valued and thick data as well as quantitative indicators) can be then used to tackle a wide variety of planning problems, including focused prediction, classification and anomaly detection, among others. Furthermore, since data and services are the most important asset that a business owns, and in today's climate of data security and stewardship it's more important than ever to trace how that data was produced and the journey it has undertaken through the workflow of services leading to its present state. What we need in this direction is a strong data and services governance platform. This platform represents another architectural layer (seventh level) to the JDL model where the layout of the system is developed as a suite of workflows of small services, each running in its own process and communicating with lightweight mechanisms. The benefits of level 7 are many, ranging from an increase in development productivity, to better business-IT alignment, agility, scalability, and technology flexibility. Figure 2 illustrate a schematic view of our proposed extended JDL model with addition of the two new levels of 6 and 7 .

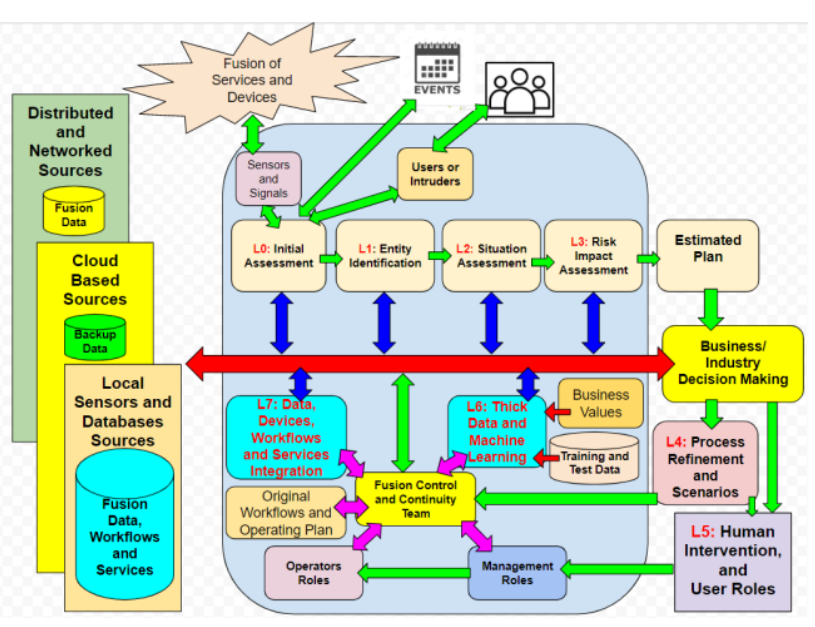

Figure 2: The Extended JDL Model for Extreme Automation and Healthcare Interoperability and Continuity.

\section{Applying the Extended JDL Model}

A number of technologies (e.g. Web Services and Agent technologies) have proposed the use of JDL data fusion model to enhance the capabilities of the network and aid in the development of situational awareness for the enforcement of business continuity, interoperability and scalability. In one hand, the Web service technology is used to support interoperability but it is still not able to support the dynamism and the autonomy aspects. In the other hand, On the other hand, agent-based modeling technology is an emerging field that can help understand business dynamics where a set of agents is used to represent various actors, imbued with simple decision-making rules, in an environment where agents interact following rules and limitations present in the real world. However, hurdles still exist in agent technology universal adoption because of the diversity of agent communication standards. For example, solving agent interoperability from a protocol and connectivity perspective is straightforward: Either using TCP/IP and/or Ethernet. However, when we peel back the layers of agent system interoperability, we eventually reach a roadblock in the form of software. How do developers get the software stack from a programmable logic controller (PLC) or programmable automation controller (PAC) from vendor A to communicate with the predictive analytics software from vendor B? For example, how would a developer move data from an automation controller into a cognitive analytics application like the IBM Watson IoT platform? More importantly, how does the developer do that without countless development cycles or hours of manpower configuring and troubleshooting complex middleware? The objective of extreme automation and healthcare interoperability is to connect sensors, industrial devices, computing systems and online services and applications together to provide plant and enterprise personnel with actionable information and cut costs. Today's such environments are changing, evolving, expanding. One need a 
single source for integrated solution tailored to the business specific workflow automation needs. The solution needs to enforce several services like:

$>$ Add new automation workflow across multiple the business ecosystem

$>$ Accelerate production, improve communications with clients, production personnel, management, improve quality, control and maximize your team's productivity.

$>$ Automate workflow retrieval and reception

$>$ Automate a wide range of third-party applications and integrate them within specified workflows

$>$ Accelerate all workflow synthesis and processing

Workflows capture the state of a business process and enable state transitions when a trigger is received. While disparate devices are turning into connected devices, there also arises a need to automate the workflows. Finding repetitive tasks and automating them will help businesses to focus on their core activities. Workflow automation also improves efficiency of the business processes and their management. Hence adopting a workflow methodology provides a robust means of describing applications consisting of control and data dependencies along with the logical reasoning necessary for distributed execution.

Designed to simplify extreme automation development, many workflow sandboxes deliver a complement of software components needed to move data from end nodes to the cloud and deliver information to end users. Workflows offer developers a simple but flexible programming model at a level of abstraction closer to the domain-specific activities that they seek to perform. However, languages for describing workflows tend to be highly complex, or specialized towards a particular domain, or both. There are a large number of workflow systems designed to work in various scientific domains, including support for the Internet of Things (IoT). One such workflow system is Node-RED ${ }^{1}$, which is originally designed to bring workflow-based programming to IoT and extreme automation. However, the majority of workflow systems including Node-RED, are designed to operate in a fixed networked environment, which rely on a central point of coordination in order to manage the workflow [18]. This paper presents ExtremeFlow: an approach to workflow programming which combines the advantages of a declarative workflow description and web services programming. The workflow execution model of ExtremeFlow is based on a formal model of computation over web services. The execution environment is implemented on the basis of a widely adopted runtime platform node.js.

\section{The ExtremeFlow Approach}

In the early days, the Web was mainly used to publish information. Then, application servers were used to offer services to human customers. We now witness development

\footnotetext{
${ }^{1}$ https://nodered.org/
}

of the Services Web where the services can be accessed programmatically and application servers collaborate with each other, typically using simple protocols like NodeJS. The Services Web evolves out of the desire to perform transactions in an open and automated environment with ubiquitous services, rather than using other mechanisms such as EDI or manual processing. The Services Web environment typically exhibits the following characteristics [19]:

- Web Services are black-box components that encapsulate behavior.

- Web Services interact using NodeJS over HTTP thus providing wrappers for interoperation between technology specific components.

- Web Services can be discovered at run-time for dynamic binding.

- Several Web Services can be orchestrated to perform a series of functions in a workflow. Web Services are units of extremely low coupling; they communicate with each other with low dependency on the other party.

However, composing web services into a coherent application can be a tedious and error prone task when using traditional textual scripting languages like WSDL [20]. As an alternative, complex interactions patterns and data exchanges between different Web services can be effectively modeled using a visual language. In this paper we present the design of the ExtremeFlow composition ecosystem. ExtremeFlow uses visual composition that has been fully implemented in a development environment for Web service composition with usability features emphasizing rapid development and visual scalability. The ExtremeFlow environment provides an integrated toolkit to manage the whole lifecycle of a workflow composition in any business (Figure 3 ).

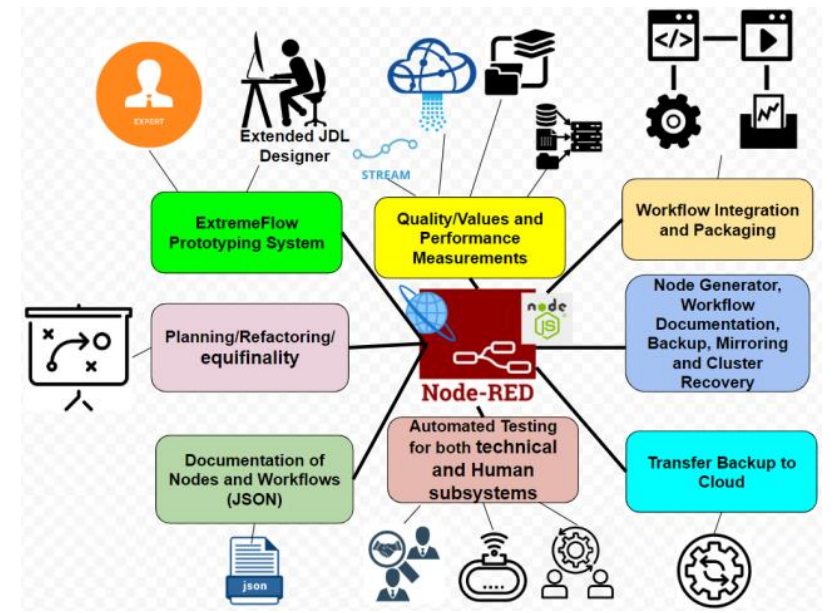

Figure 3: ExtremeFlow Architecture for Implementing the Extended JDL Workflow Model.

In order to manage and fuse these sensor and services feeds, an architecture is required that integrates the services in a loosely coupled way to support decentralized discovery and execution. This loosely coupled nature ensures that 
existing services and resources can be quickly set up to be discovered and take part in query responses without having to be re-written from the ground up. For this reason, the ExtremeFlow needs to have component which is the NodeRed IFTTT Broker. This new component provides library methods to allow services to send and receive IFTTT $^{2}$ Triggers and Actions is a web-based service to create chains of simple conditional statements, called applets. An applet is triggered by changes that occur within other web services such as Gmail, Facebook, Telegram, Instagram, or Pinterest. Figure 4 illustrate the usage of the Node-Red IFTTT broker in developing and composing workflows in the healthcare paradigm.

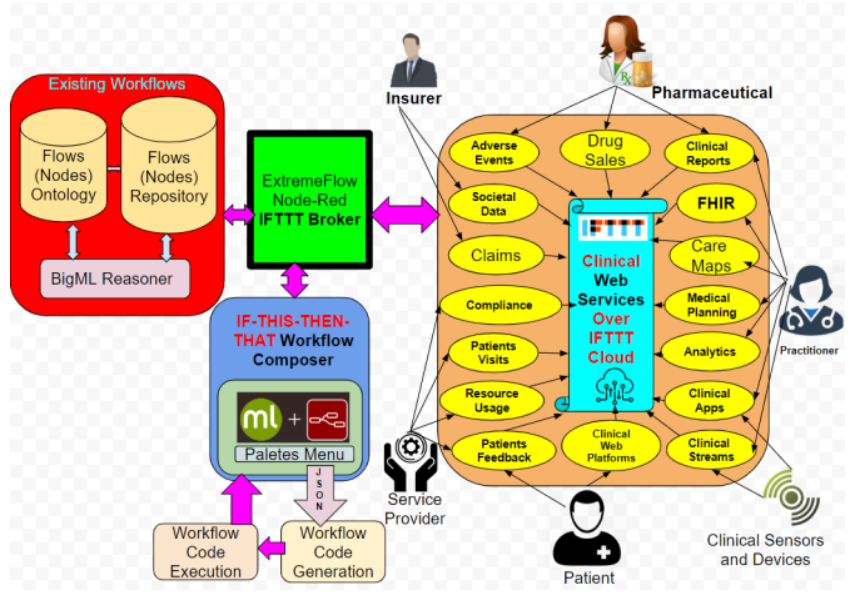

Figure 4: Implementing the ExtremeFlow using the IFTTT Broker for eHealth workflow applications.

The composition process using the IFTTT broker begins with the Flow Repository, where Web services can be imported as reusable components. Any user can search the repository with the help of ontology and a reasoner, select a set of existing services and drag them into the workflow composer. Then, the new workflow can be modified by adding new nodes from the palate's menu or from the web services published over the cloud. This operation is partially automatic, since the editor can bind parameters with matching names. To get an overview over the order of execution of the tasks and add additional constraints, the user may view and edit the workflow anytime. Once all of the services have been connected the new process may be compiled and uploaded to an ExtremeFlow runtime environment for execution. The user may interact with a running process or its tasks, and abort, pause, continue, and restart them at will. More than one copy of a process may be run concurrently. Once a process has completed its execution, the user may access the content of all parameters as well as measurements about the execution time of each task, until the process is explicitly deleted from the system.

${ }^{2}$ https://ifttt.com/
The IFTTT workflow composer uses a flexible domain specific language (DSL) to solve a range of problems in the eHealth application domain. Even though a domain specific language is limited in its usage outside the domain, it can be used to write efficient code to solve problems in the target domain [21, 22]. There has been a range of DSLs (e.g. Tasker, IFTTT) for heterogeneous systems like those for IoT, to enhance the programmability or simplify the process of wiring different components of an IoT system [23]. This type of DSLs allows users to create workflows with "triggers" and "actions." The "IF THIS" keyword is used to identify the triggers and "THEN THAT" is to identify the actions part. The trigger is activated by changes that occur within other web services such as FITBIT steps or an email from Gmail. Upon activation you may assign an action like pushing a reminder notification about your achieved steps:

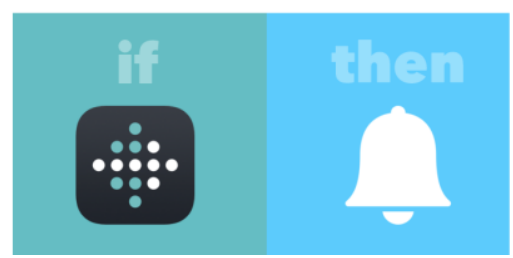

and you may chain this part of the workflow with another part like if a notification from Gmail arrived then you will add it to your TODO list:

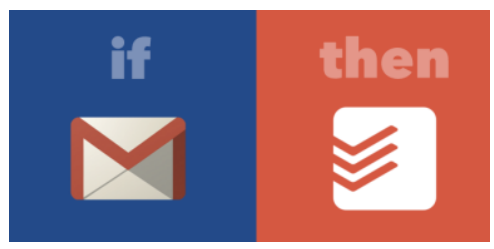

The automations are accomplished via Node-Red recipe with IFTTT $^{3}$ integration - which are sort of like macros that connect multiple apps and devices to run automated tasks. The recipe employs the following concepts:

- Triggers are the "this" part of the recipe. They are the items that trigger the action. For example, from an RSS feed, you can receive a notification based on a keyword or phrase.

- Actions are the "that" part of the recipe. They are the output that results from the input of the trigger.

- $\quad$ Recipes are the predicates made from Triggers and Actions. For example, if you like a picture on Instagram (trigger), an ExtremeFlow app can send the photo to your Dropbox account (action).

- Ingredients are basic data available from a triggerfrom the email trigger, for example; subject, body, attachment, received date, and sender's address.

As an example, to illustrate using our ExtremeFlow composer to detect the presence of patients wearing a Fitbit

${ }^{3}$ https://ifttt.com/ 
Flex ${ }^{4}$ near a Bluetooth (BLE) scanner ${ }^{5}$. In the composer we will need to create an initial flow where we will drag one IFTTT node (now called event1):

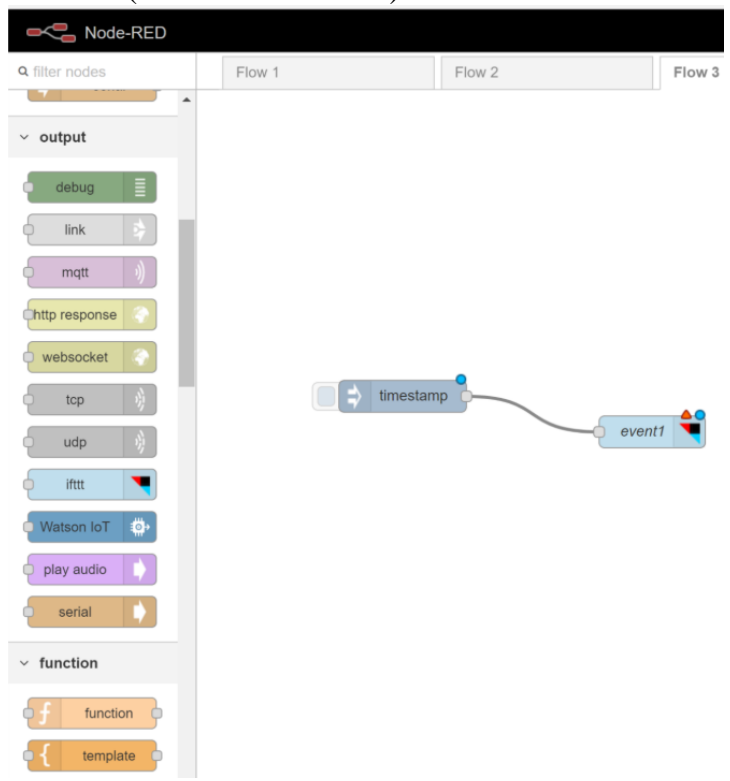

However, we will need to define by clicking on the IFTTT event 1 where we will be directed to

https://ifttt.com/my_applets to create a new IFTTT applet:

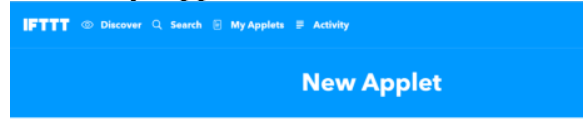

\section{if + this then that}

By choosing the BLE Scanner this trigger will be set to this service and we will need to set the action required when the trigger will be activated:

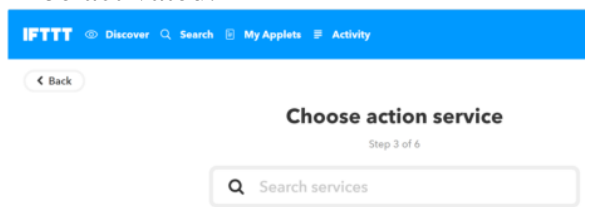

Now a complete workflow will look like this:

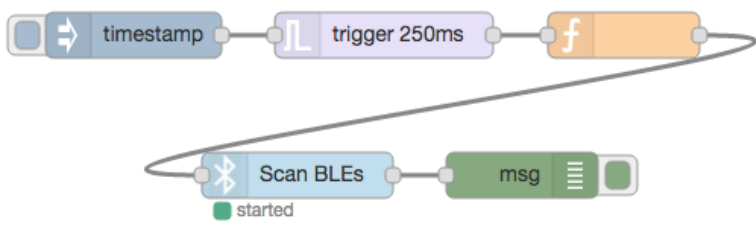

We can inject a message to scan for the Fitbit Bluetooth availability every 10 seconds:

\footnotetext{
${ }^{4}$ https://www.fitbit.com/en-ca/flex2

${ }^{5} \mathrm{http} / / /$ developers.sensetecnic.com/article/detect-presence-using-afitbit-node-red-and-fred/
}

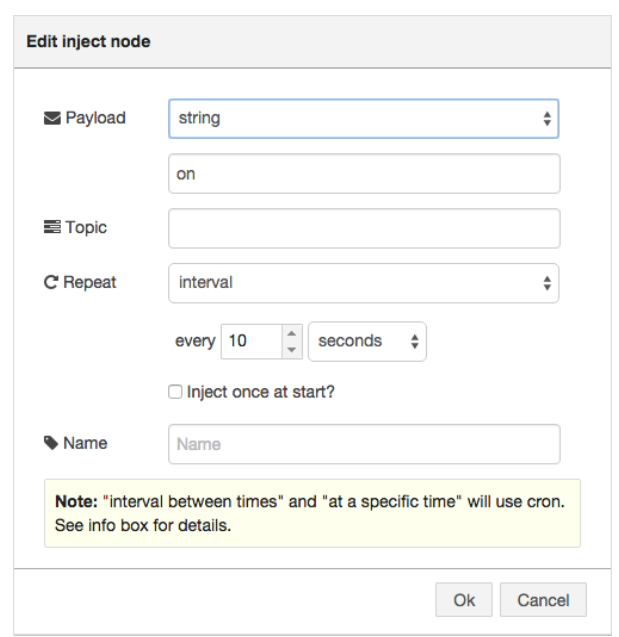

Once this is done, bring a Fitbit flex in range to the computer and deploy the flow. If all is well you should should see output similar to Listing 1 in the debug pane when it discovers devices.

\{ "payload": \{ "peripheralUuid":

"88e1ab7bcfa4493dbaf16c55e69a4881",

"localName": "Flex" \}, "peripheralUuid":

"88e1ab7bcfa4493dbaf16c55e69a4881",

"localName": "Flex", "detectedAt":

1452023547186, "detectedBy": "myhost.local",

"advertisement": \{ "localName": "Flex",

"txPowerLevel": -6, "serviceData": [ \{ "uuid":

"180a", "data": [ 7,4 ] \} ], "serviceUuids": [

"adab1fb86e7d4601bda2bffaa68956ba" ] \}, "rssi": -89, "_msgid": "a017136d.5fe8f" \}

\section{CONCLUSION}

We tested our ExtremeFlow composer on a Google Pixel 3 running Android 9. In every application domain, interoperability between various services comprises the integration of computation, software, networking, and physical processes. Consequently, interoperability models required an increasing support for hybrid and heterogeneous models, networking, services and time synchronization. To assist developers and users alike in designing such systems, we have developed a prototype for composing workflows based on incorporating an IFTTT Broker (IF-THIS-THENTHAT) Domain-Specific Language (DSL) that comes with fully-automated Node-Red and IFTTT tool support. Our ExtremeFlow composer include support for: (i) interactive Node-Red model description with input validation; (ii) the computation of possible operation modes of subsystems and parts; and, (iii) checking the adherence to requirements for various design alternatives and finding the near optimal designs given these requirements. Moreover, the generated workflow models provide visualizations throughout the toolchain which help design engineers to better understand the implications of design decisions and communicate them to stakeholders. The ExtremeFlow composer has been applied to the healthcare domain. We are currently 
experimenting to extend the ExtremeComposer to provide smart analytic capabilities through integrating the Node-Red with the $\mathrm{BigML}^{6}$ services.

\section{ACKNOWLEDGMENT}

This research is a preprint extracts from the NSERC Discovery Grants 2020 Proposals of the two authors.

\section{REFERENCES}

[1] M. E. Liggins, C. Y. Chong, I. Kadar et al., "Distributed fusion architecture and algorithms for target tracking," Proceedings of IEEE. pp. 95-107, 1997.

[2] Zhao, Baizhong, and Frederick Steier. "Effective Computer Integrated Manufacturing (CIM) implementation using Socio-Technical Principles." Industrial Management 35, no. 3 (1993): 27.

[3] Klaus Schwab, The Fourth Industrial Revolution: what it means, how to respond, World Economiv Forum, 14 Jan 2016, Available Online: https://www.weforum.org/agenda/2016/01/the-fourth-industrial-revolutionwhat-it-means-and-how-to-respond/

[4] Davies, Robert, Tim Coole, and Alistair Smith. "Review of sociotechnical considerations to ensure successful implementation of Industry 4.0." Procedia Manufacturing 11 (2017): 1288-1295.

[5] Liu, Kecheng. Semiotics in information systems engineering. Cambridge University Press, 2000.

[6] Cordeiro, José, and Joaquim Filipe. "The Semiotic Pentagram Framework--A Perspective On The Use of Semiotics within Organizational Semiotics." In Proceedings of the 7th International Workshop on Organisational Semiotics. 2004.

[7] Liu, Kecheng. "Requirements reengineering from legacy information systems using semiotic techniques." Syst Signs Actions Int J Commun Inf Technol Work 1, no. 1 (2005): 38-61.

[18] Alan Steinberg, Christopher Bowman and Franklin White, "Revisions to the JDL Data Fusion Model," March 1999. Proc. SPIE Vol. 3719, p. 430-441, Sensor Fusion: Architectures, Algorithms, and Applications III, Belur V. Dasarathy; Ed.

[19] James Llinas, Christopher Bowman, Galina Rogova, Alan Steinberg, Ed Waltz and Franklin White, "Revisiting the JDL Data Fusion Model II," December 2004. Available Online:

https://pdfs.semanticscholar.org/b183/008f9d63252bd4cc4438cf0bf744bc8 e995d.pdf

[20] B. Dasarathy, "Decision Fusion Strategies in Multisensor

Environments “, IEEE Transactions on Systems, Man, and Cybernetics, pp 1140-1154, vol. 21, 1991.

[11] M. Bedworth and J. Obrien, "The Omnibus Model: A New model of data fusion?", AES Magazine, April 2000.Available Online:

https://pdfs.semanticscholar.org/7595/1a01b0c603774ed99e57d9b427ce55 741020.pdf

[12] Kadar, I, "Perceptual Reasoning in Adaptive Fusion Processing," SPIE, 2002, Available

Online:https://www.spiedigitallibrary.org/conference-proceedings-ofspie/4729/0000/Perceptual-reasoning-in-adaptive-fusionprocessing/10.1117/12.477619.short

[13] Asuncion, C.H. and Van Sinderen, M.J., 2010, September. Pragmatic interoperability: A systematic review of published definitions. In IFIP International Conference on Enterprise Architecture, Integration and Interoperability (pp. 164-175). Springer, Berlin, Heidelberg.

[14] Liggins, Martin E.; Hall, David L.; Llinas, James (2008). Multisensor Data Fusion, Second Edition: Theory and Practice (Multisensor Data Fusion). CRC. ISBN 978-1-4200-5308-1.

[15] Mark Meloon, What is an example of data fusion? How does it help in big data? Is it data analysis technique?, Sep 19, 2014, Available Online: https://www.quora.com/What-is-an-example-of-data-fusion-How-does-ithelp-in-big-data-Is-it-data-analysis-technique

[16] Erik Blasch, One decade of the Data Fusion Information Group (DFIG) model, Proceedings Volume 9499, Next-Generation Analyst III; 94990L (2015) https://doi.org/10.1117/12.2176934

Event: SPIE Sensing Technology + Applications, 2015, Baltimore,
Maryland, United States

[17] Fiaidhi, Jinan, and Sabah Mohammed. "Thick Data: A New Qualitative Analytics for Identifying Customer Insights." IT Professional 21.3 (2019): 4-13.

[18] Simpkin, Christopher, Ian Taylor, Daniel Harborne, Graham Bent, Alun Preece, and Ragu K. Ganti. "Dynamic Distributed Orchestration of Node-RED IOT Workflows Using a Vector Symbolic Architecture." In 2018 IEEE/ACM Workflows in Support of Large-Scale Science (WORKS), pp. 52-63. IEEE, 2018.

[19] Ganesarajah, Dinesh, and Emil Lupu. "Workflow-based composition of web-services: a business model or a programming paradigm?." In Proceedings. Sixth International Enterprise Distributed Object Computing, pp. 273-284. IEEE, 2002.

[20] Pautasso, Cesare, and Gustavo Alonso. "Visual composition of web services." In IEEE Symposium on Human Centric Computing Languages and Environments, 2003. Proceedings. 2003, pp. 92-99. IEEE, 2003. [21] van den Berg, Freek, Vahid Garousi, Bedir Tekinerdogan, and Boudewijn R. Haverkort. "Designing cyber-physical systems with aDSL: A domain-specific language and tool support." In 2018 13th Annual Conference on System of Systems Engineering (SoSE), pp. 225-232. IEEE, 2018.

[22] Nägele, Thomas, and Jozef Hooman. "Rapid Construction of Cosimulations of Cyber-Physical Systems in HLA using a DSL." In 2017 43rd Euromicro Conference on Software Engineering and Advanced Applications (SEAA), pp. 247-251. IEEE, 2017.

[23] Quirk, Chris, Raymond Mooney, and Michel Galley. "Language to code: Learning semantic parsers for if-this-then-that recipes." In Proceedings of the 53rd Annual Meeting of the Association for Computational Linguistics and the 7th International Joint Conference on Natural Language Processing (Volume 1: Long Papers), pp. 878-888. 2015.

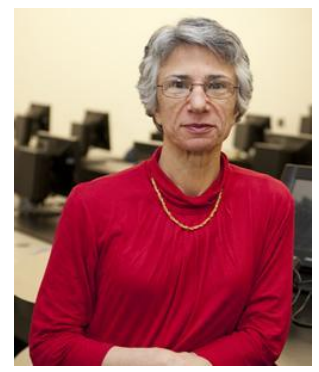

\section{Jinan Fiaidhi}

Dr. Fiaidhi is a full professor of Computer Science and the Graduate Coordinator of the Biotechnology $\mathrm{PhD}$ program at Lakehead University. She is an adjunct research professor at the University of Western Ontario and the editor in chief of IGI Global International Journal of Extreme Automation and Connectivity in Healthcare. She is also the chair of Big Data for eHealth with the IEEE ComSoc. Contact her at jfiaidhi@lakeheadu.ca.

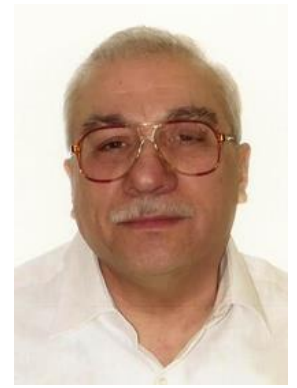

\section{Sabah Mohammed}

Dr. Mohammed is a full professor of Computer Science and Professional Software Engineer. $\mathrm{He}$ is an adjunct research professor at the University of Western Ontario and the editor in chief of IGI Global International Journal of Extreme Automation and Connectivity in Healthcare. He is also the chair of Smart and Connected Health with the IEEE ComSoc. Contact him Sabah.mohammed@lakeheadu.ca. 